\title{
Eficiência da Eletroejaculação de Morfotipos Machos do Camarão-de-água-doce Macrobrachium rosenbergii ${ }^{1}$
}

\author{
Rubens Sterental Goldberg², Lídia Miyako Yoshii Oshiro³
}

\begin{abstract}
RESUMO - O objetivo deste trabalho foi avaliar a aplicabilidade da técnica de eletroejaculação para manejos de inseminação artificial, bem como o potencial ejaculatório de cada um dos tipos morfológicos de machos adultos do Macrobrachium rosenbergii. Estímulos elétricos foram aplicados em 48 exemplares de camarão-de-água-doce, recém capturados em viveiros de engorda, para melhor compreensão do comportamento reprodutivo de cada um: os de quela azul (BC), sexualmente ativos e dominantes; os de quela laranja (OC), sexualmente inativos; e os pequenos (SM), com comportamento oportunista, os quais somente realizam a cópula, caso a corte da fêmea tenha sido previamente realizada por um macho BC. Não houve diferença entre as freqüências de ejaculação observadas para machos OC e BC, que foram 85 e $100 \%$, respectivamente, demonstrando que os primeiros possuem potencial fisiológico para a cópula. A freqüência de $46,2 \%$, nos testes de eletroejaculação bem sucedidos para os indivíduos SM, foi mais baixa em relação aos outros dois morfotipos. A frequiência de 79,2\% de ejaculações, obtida entre o total de animais usados, demonstrou o potencial dessa técnica para a obtenção de espermatóforos em quantidade suficiente para a manutenção de fêmeas ovadas em épocas de baixas temperaturas.
\end{abstract}

Palavras chave: ejaculação, inseminação artificial, Macrobrachium rosenbergii, morfotipo, espermatóforo

\section{Electroejaculation Efficiency of Male Morphotypes of the Freshwater Prawn Macrobrachium rosenbergii}

\begin{abstract}
The objective of this work was to evaluate the electroejaculation applicability for artificial insemination as well as the ejaculatory potential of each Macrobrachium rosenbergii adult male morphotype. Electrical stimuli were applied to 48 newly pond - reared individuals of freshwater prawn for a better comprehension of each one's reproductive behavior: the blue claw males (BC), sexually active and dominant; the orange claw (OC), sexually inactive, and the small ones (SM), with opportunist behavior, succeeding to copulate only if a BC male has made the courtship first. There was no difference among the ejaculation frequencies for OC and BC males, which were 85 and $100 \%$, respectively, demonstrating that the former morphotype has physiological potential for mating. The frequency of $46.2 \%$ of successful eletroejaculation tests observed for SM individuals was lower in relation to the two other morphotypes. The frequency of $79.2 \%$ of ejaculations observed for all used animals demonstrated the technique potential for the attainment of a sufficient amount of spermatophores prior to the maintenance of gravid females during cold temperature seasons.
\end{abstract}

Key Words: ejaculation, artificial insemination, Macrobrachium rosenbergii, morphotype, spermatophore

\section{Introdução}

O conhecimento dos sistemas endocrinológico e genético do camarão Macrobrachium rosenbergii constitui subsídio para a indução de taxas de crescimento mais elevadas destes animais. A implicação desses sistemas no comportamento reprodutivo da espécie, por sua vez, pode favorecer a elaboração de técnicas de manejo reprodutivo com aplicação na aqüicultura.

Dentro desse contexto, a origem da diferenciação dos machos adultos da espécie entre três tipos morfológicos e o comportamento de cada um têm sido amplamente estudados (RA'ANAN et al., 1991; RA'ANAN e COHEN, 1985; e DANIELS et al., 1995).

Os morfotipos correspondem a etapas ontogênicas do desenvolvimento do macho e são seqüencialmente: machos de quela azul (BC), machos de quela laranja (OC) e machos pequenos (SM).

Os machos BC são maiores, além de possuírem domínio territorial e serem sexualmente ativos; os machos OC, cujo tamanho corporal médio é ligeiramente menor, são sexualmente inativos na presença do morfotipo BC; e os indivíduos SM, cujas quelas apresentam pouca pigmentação e coloração indefinida, são os menores dentro da população de machos,

\footnotetext{
1 Parte da Tese de Mestrado do primeiro autor, apresentada ao Instituto de Zootecnia da Universidade Federal Rural do Rio de Janeiro - Km 47 da antiga rodovia Rio-São Paulo, Seropédica- RJ CEP: 23.851-970. Instituição financiadora do projeto: UFRRJ.

${ }^{2}$ Biólogo, Mestre em Zootecnia. E-mail: rubigold@zipmail.com.br

3 Professor Adjunto do IZ da UFRRJ. E-mail: dimas@ibm.net
} 
apresentando comportamento oportunista em relação à cópula, uma vez que se aproveitam da corte realizada pelos machos $\mathrm{BC}$ para se colocarem entre estes e as fêmeas e, então, acasalarem (RA'ANAN e COHEN, 1985).

A transformação de machos SM em OC compreende formas intermediárias, adquiridas ao longo de ciclos intermudas. Ao atingirem o morfotipo OC, os machos continuam a passar por mudas de crescimento, entretanto, a transformação morfotípica para BC ocorrerá, sem formas intermediárias, por intermédio de uma única muda (KURIS et al., 1987).

A partir da fase inicial de macho SM, o animal pode manter seu morfotipo, o que possibilitará uma cópula oportunista, embora com poucas chances de efetivação, ou transformar-se em macho OC e subseqüentemente em macho BC (RA'ANAN e COHEN,1985). Portanto, em condições naturais, os animais do morfotipo OC jamais cortejam fêmeas e a transformação morfotípica para a etapa seguinte ocorre apenas eventualmente, por parte somente dos exemplares maiores, quando o macho OC se confronta com um BC de menor tamanho ou fragilizado por doença (RA'ANAN e COHEN, 1985).

A técnica de eletroejaculação constitui a forma mais difundida de obtenção do espermatóforo em crustáceos, por ser mais rápida e fornecer o espermatóforo na forma como ocorre a fertilização na natureza. Essa técnica foi utilizada pela primeira vez em M. rosenbergii por SANDIFER e LYNN (1980).

A cópula entre os animais desta espécie ocorre com a deposição do espermatóforo na placa espermática da fêmea, localizada na região ventral externa do cefalotórax, ou seja, entre os terceiro e quarto pares de pereiópodos. O contato entre os gametas ocorre por meio da expulsão dos óvulos pelos poros genitais, que então vão ao encontro dos espermatozóides contidos no espermatóforo, promovendo, dessa forma, a fecundação (SANDIFER e SMITH, 1979).

HARRIS e SANDIFER (1986) testaram comparativamente as taxas de fecundidade de espermatóforos de $M$. rosenbergii inseminados por intermédio de cópula e inseminação, com uso do sêmen obtido por eletroejaculação, não tendo sido verificado diferença significativa entre ambos os tratamentos. Os mesmos autores verificaram ainda os efeitos danosos dos estímulos elétricos nos animais, manifestados por meio de lesões em suas vias espermáticas.

As técnicas de manejo de inseminação artificial, portanto, constituem alternativas para o aproveitamento de machos com bom potencial genético, porém incapazes de desempenhar comportamento voltado para o acasalamento, como os do morfotipo OC ou aqueles em manejos de hibridização. Além desses fatores, esses manejos poderão viabilizar o suprimento de larvas em épocas de baixa temperatura.

O objetivo deste trabalho foi avaliar a aplicabilidade da técnica de eletroejaculação para manejos de inseminação artificial, bem como o potencial ejaculatório de cada um dos tipos morfológicos de machos adultos de $M$. rosenbergii para melhor compreensão de seu comportamento reprodutivo.

\section{Material e Métodos}

Os exemplares do camarão-de-água-doce $M$. rosenbergii foram coletados de viveiros de engorda do Posto de Aqüicultura da Universidade Federal Rural do Rio de Janeiro (UFRRJ).

Foram selecionados, aleatoriamente, 48 indivíduos machos, sexualmente maturos, com idade de oito meses, distribuídos de acordo com os seguintes morfotipos: 15 machos BC, 20 machos OC e 13 machos SM, com peso e comprimento de $55,8 \pm 11,5 \mathrm{~g}$ e $16,6 \pm 1,1 \mathrm{~cm} ; 40,3 \pm 7,6$ g e $15,5 \pm 1,0 \mathrm{~cm}$ e $21,4 \pm 5,5$ g e $12,7 \pm 1,5 \mathrm{~cm}$, respectivamente. Cada exemplar foi submetido a um único teste com estímulos elétricos em corrente contínua de 9 volts (V), aplicado por meio de bateria, com auxílio de eletrodos.

A aplicação dos estímulos elétricos nos animais foi feita por meio de contínuos toques dos eletrodos, com duração de aproximadamente um segundo, ao longo de até um minuto, próximo à região do par de poros genitais, localizadona base do quinto par de pereiópodos.

A corrente elétrica induziu as contrações musculares, que provocaram a expulsão do espermatóforo armazenado na ampola terminal do canal deferente.

Os dois eletrodos foram contatados inicialmente a cada uma das coxas do quinto par de pereiópodos. Quando este procedimento não foi suficiente para a obtenção da ejaculação, nova tentativa foi executada com o posicionamento de um deles na coxa e o outro no tórax, entre os dois poros genitais. Em caso de novo fracasso, foi tentado por último a colocação de um dos eletrodos no primeiro pleonito ou segmento abdominal e o segundo em uma das coxas. Estas posições basearam-se no trabalho de AIKEN et al., (1984), quando estudaram lagostas da espécie Homarus americanus, e HARRIS e SANDIFER (1986), trabalhando com M. rosenbergii.

Os resultados satisfatórios para os testes de eletroejaculação consideraram a obtenção de pelo me- 
nos um espermatóforo. As frequiências de ejaculações obtidas para cada morfotipo foram comparadas por intermédio de testes de qui-quadrado, em tabelas de contingência 2 × $2 \quad(\mathrm{P}<0,05)$ com correção de Yates.

\section{Resultados e Discussão}

Entre os 48 testes de eletroejaculação realizados, foi obtido sucesso em 38, correspondendo a 79,2\% do total. Este resultado permite avaliação positiva do potencial de aplicação desta técnica para a obtenção de espermatóforos em inseminações artificiais.

A porcentagem de testes de eletroejaculação, realizados com sucesso neste experimento, foi superior à encontrada por GOLDBERG e ANNIBAL (1998), que obtiveram 50\% de ejaculações, após aplicação de estímulos de 4,5 V em dose única, com machos BC de $M$. rosenbergii recém despescados de viveiros de engorda. HARRIS e SANDIFER (1986) obtiveram freqüências de ejaculação entre 81,1 e $100 \%$ para animais da mesma espécie, mantidos em cativeiro, submetidos a diferentes freqüências de repetição de estímulos de $4 \mathrm{~V}$ ao longo de 29 dias.

A aplicação de um único teste de eletroejaculação em animais recém-despescados de viveiros de engorda, neste trabalho, propôs a utilização de um universo com maior disponibilidade de machos que a capacidade de armazenagem dos animais em laboratório, permitindo, portanto, a obtenção de maior quantidade de espermatóforos e a fertilização de fêmeas por meio do sêmen previamente estocado, ao invés de recém coletado de machos mantidos em cativeiro.

A estocagem de espermatóforos flexibiliza os manejos de inseminação artificial, uma vez que requer espaço menor, assegura o acesso ao material genético masculino, independentemente da disponibilidade de machos com potencial para fornecer espermatóforos mediante estímulos elétricos, e permite sua estocagem por um período mais extenso, pois o confinamento de machos pode acarretar queda da qualidade de seus materiais genéticos. TRUJILLO e LAWRENCE (1987) detectaram a ausência de espermatozóides em espermatóforos de camarões marinhos Penaeus setiferus após seis semanas em laboratório. HARRIS e SANDIFER (1986), embora não tenham verificado decréscimo na taxa de fertilização de fêmeas, para os espermatóforos coletados ao longo de até 29 dias, constataram diminuição significativa no volume do ejaculado, o que a longo prazo pode constituir fator limitante para eventuais inseminações.
CHOW et al. (1985) foram bem sucedidos na obtenção de larvas de $M$. rosenbergii, a partir de fêmeas inseminadas com espermatóforos armazenados em vapor de nitrogênio líquido durante 30 dias. GOLDBERG (1998), por intermédio da mesma técnica de estocagem, com a mesma espécie, obteve viabilidade espermática média de $64,8 \%$ após 60 dias.

A maior parte dos testes de eletroejaculação proporcionou a expulsão do espermatóforo de apenas um dos poros genitais. Entretanto, embora não existam estudos específicos quanto à quantidade mínima de espermatozóides necessários para a inseminação de fêmeas de $M$. rosenbergii, SANDIFER e SMITH (1979) e SANDIFER e LYNN (1980) obtiveram sucesso na inseminação de fêmeas com apenas o uso de fragmentos do ejaculado.

Tomando-se por base os dados obtidos por WICKINS e BEARD (1974) para M. rosenbergii (30.000 ovos por desova e frequiência média de desovas de $80 \%$ ), a utilização dos espermatóforos obtidos no presente estudo em manejo artificial seria capaz de proporcionar a inseminação de 38 fêmeas que produziriam 912.000 larvas .

As frequiências de testes de eletroejaculação bem sucedidos para os machos $\mathrm{BC}$, OC e SM foram, respectivamente, de 100,85 e $46,15 \%$. Os testes de Qui-quadrado realizados para estes resultados não apontaram diferença significativa entre as freqüências obtidas para os exemplares de machos BC e OC, conforme demonstrado na Tabela 1. Os machos SM, por sua vez, tiveram resultados estatisticamente diferentes dos morfotipos $\mathrm{BC}$ e OC, conforme demonstrado, respectivamente, nas Tabelas 2 e 3.

Estes dados são insuficientes para comparação entre os potenciais reprodutivos dos três tipos de macho, uma vez que, conforme observado ao longo

Tabela 1- Comparação das freqüências de eletroejaculação, pelo teste do qui-quadrado $\left(\mathrm{X}^{2}\right)$, de machos de $M$. rosenbergii de diferentes morfotipos

Table 1 - Comparison of the electroejaculation frequencies by the chi-square test $\left(X^{2}\right)$ of $M$. rosenbergii means of different male morphotypes

\begin{tabular}{lccc}
\hline $\begin{array}{l}\text { Morfotipo } \\
\text { Morphotype }\end{array}$ & $\begin{array}{c}\text { Ejaculação } \\
\text { Ejaculation }\end{array}$ & $\begin{array}{c}\text { Sem ejaculação } \\
\text { No ejaculation }\end{array}$ & Total \\
\hline BC & $15(13,71)$ & $0(1,29)$ & 15 \\
OC & $17(18,28)$ & $3(1,71)$ & 20 \\
Total & 32 & 3 & 35 \\
\hline
\end{tabular}

$x^{2}=3,06 \quad p>0,05$

$\mathrm{BC}$ - macho de quela azul; OC - macho de quela laranja.

$B C$ - blue claw male; $O C$ - orange claw male. 
Tabela 2 - Comparação das freqüências de eletroejaculação, pelo teste do qui-quadrado $\left(\mathrm{X}^{2}\right)$, de machos de $M$. rosenbergii de diferentes morfotipos

Table 2 - Comparison of the electroejaculation frequencies by means of the chi-square test $\left(X^{2}\right)$ of $M$. rosenbergii different male morphotypes

\begin{tabular}{lccc}
\hline $\begin{array}{l}\text { Morfotipo } \\
\text { Morphotype }\end{array}$ & $\begin{array}{c}\text { Ejaculação } \\
\text { Ejaculation }\end{array}$ & $\begin{array}{c}\text { Sem ejaculação } \\
\text { No ejaculation }\end{array}$ & Total \\
\hline BC & $15(11,25)$ & $0(3,75)$ & 15 \\
SM & $6(9,75)$ & $7(3,25)$ & 13 \\
Total & 21 & 7 & 28 \\
\hline
\end{tabular}

$\mathrm{x}^{2}=10,86^{*} ; \mathrm{P}<0,05$

BC- macho de quela azul; SM- macho pequeno.

$B C$ - blue claw male; $S M$ - small male.

do experimento, os animais do morfotipo SM demonstraram maior desgaste após os estímulos elétricos, o que pode ser indício de correlação entre as freqüências de ejaculação obtidas para cada morfotipo e eventual diferença da capacidade de reação aos estímulos elétricos, em função de seus tamanhos corporais. Entretanto, ficou demonstrado claramente que cada um possui potencial para produzir espermatóforos e ejacular.

A ausência de comportamento reprodutivo por parte dos machos OC, descrito por RA' ANAN e COHEN (1985), ocorre, aparentemente, devido a um padrão comportamental utilizado como estratégia reprodutiva, no sentido de evitar confrontos com os machos dominantes BC: Tal padrão está associado a um ritmo de crescimento relativamente mais rápido (RA'ANAN et al. 1991), condicionado pela ação do hormônio da glândula androgênica (SAGI et al., 1990), acelerando a transformação morfotípica para macho BC.

O comportamento reprodutivo oportunista dos machos SM torna-se mais conveniente para estes animais, uma vez que a maior mobilidade destes indivíduos (RA'ANAN e COHEN, 1985) lhes confere melhores chances de acasalamento desta forma do que por intermédio de eventual transformação morfotípica para macho BC.

O ritmo lento de crescimento dos indivíduos SM e BC e, concomitantemente, a baixa ocorrência de mudas, por sua vez, podem estar ligados à adaptação voltada para o acasalamento por meio da manutenção do corpo do animal com a carapaça dura, condição fundamental para que esta ocorra.
Tabela 3 - Comparação das freqüências de eletroejaculação, pelo teste do qui-quadrado $\left(\mathrm{X}^{2}\right)$, de machos de $M$. rosenbergii de diferentes morfotipos

Table 3 - Comparison of the electroejaculation frequencies by the chi-square test $\left(\mathrm{X}^{2}\right)$, of $M$. rosenbergii different male morphotypes

\begin{tabular}{lccc}
\hline $\begin{array}{l}\text { Morfotipo } \\
\text { Morphotype }\end{array}$ & $\begin{array}{c}\text { Ejaculação } \\
\text { Ejaculation }\end{array}$ & $\begin{array}{c}\text { Sem ejaculação } \\
\text { No ejaculation }\end{array}$ & Total \\
\hline OC & $17(13,94)$ & $3(6,06)$ & 20 \\
SM & $6(9,06)$ & $7(3,93)$ & 13 \\
Total & 23 & 10 & 33 \\
\hline
\end{tabular}

$x^{2}=5,64^{*} \quad p<0,05$

OC - macho de quela laranja; SM - macho pequeno.

$O C$ - orange claw male; SM - small male.

\section{Conclusões}

A aplicação em dose única da eletroejaculação possibilitou a obtenção de espermatóforos de maneira rápida, viabilizando, dessa forma, sua estocagem para manejos de inseminação artificial em fêmeas de M.rosenbergii.

O uso de estímulos de 9 volts foi eficiente na indução de ejaculação em machos do camarão-deágua-doce M.rosenbergii.

Os três tipos morfológicos comparados apresentaram potencial para a produção de sêmen e a ejaculação, sendo viáveis para a inseminação artificial.

As freqüências de ejaculação observadas para machos $\mathrm{BC}$ e OC sugerem que o potencial reprodutivo entre estes morfotipos é igual e a diferença entre seus comportamentos em relação à cópula está somente relacionada à etologia.

\section{Referências Bibliográficas}

AIKEN, D.E., WADDY, S.L., MORELAND, K. et al. 1984. Electrically induced ejaculation and artificial insemination of the american lobster Homarus americanus. J. Crust. Biol., 4:518-527.

CHOW, S., TAKI, Y., OGASAWARA, Y. 1985. Cryopreservation of the spermatophore of the fresh water shrimp Macrobrachium rosenbergii. Biol Bull., 168:471-475.

DANIELS, W.H., ABRAMO, L.R., FONDREN, M.W. et al. 1995. Effects of stocking density and feed on pond production characteristics and revenue of harvested freshwater prawn Macrobrachium rosenbergii stocked as size-graded juveniles. J. World Aquacult. Soc., 26(1):38-47.

GOLDBERG, R.S. Comparação da eficiência da eletroejaculação entre tipos morfológicos de macho e manejos de criopreservação espermática do camarão de água doce 
Rev. bras. zootec.

Macrobrachium rosenbergii. Seropédica - RJ: Instituto de Zootecnia da UFRRJ, 1998 . 61p . Dissertação (Mestrado em Zootecnia) - Universidade Federal Rural do Rio de Janeiro, 1998.

GOLDBERG, R.S., ANNIBAL, S.R.P. Eficiência da técnica de eletroejaculação para a obtenção de sêmen do camarão de água doce Macrobrachium rosenbergii. In: CONGRESSO BRASILEIRO DE ZOOLOGIA, 22, 1998, Recife, 1998. Anais... Recife: SBZ, 1998. p.87.

HARRIS, S.E.G., SANDIFER, P.A. 1986. Sperm production and the effects of the electrically induced spermatophore expulsion in the prawn Macrobrachium rosenbergii (De Man). J. Crust. Biol., 6(4):633-647.

KURIS, A.M., RA'ANAN, Z., SAGI, A. et al. 1987. Morphotypic differentiation of male Malaysian prawns, Macrobrachium rosenbergii. J.Crust. Biol., 7(2):219-237.

RA'ANAN, Z., COHEN, D. 1985. Ontogeny of social structure and population dynamics in the giant freshwater prawn, Macrobrachium rosenbergii (De Man) In: WENNER, A.M. Crustacean Issues 3, Factors in adult growth, Rotterdam, Boston. p.277-311.

RA'ANAN, Z., SAGI, A., WAX, Y. et al. 1991. Growth size rank and maturation of the freshwater prawn Macrobrachium rosenbergii: Analysis of marked prawns in an experimental population. Biol. Bull. Mar. Biol., 181(3):379-386.
SAGI, A., COHEN, D., MILNER, Y. 1990. Effect of androgenic gland ablation on morphotypic differentiation and sexual characteristics of male freshwater prawns Macrobrachium rosenbergii. Gen. Com. Endocrinol., 77(1):15-22.

SANDIFER, P.A., LYNN, J.W. 1980. Artificial insemination of caridean shrimp. In: CLARK JR., W.H., ADAMS, T.S. (Eds.). Advances in invertebrate reproduction: Elsevier North Holland. p.271-288.

SANDIFER, P.A., SMITH, T.I.J. 1979. A method for artificial insemination of Macrobrachium prawns and it's potential use in inheritance and hybridization studies. W. Mar. Soc., 10:403-418.

TRUJILLO, J.L., LAWRENCE, A.L. 1987. Observations on the decline in sperm quality of Penaeus setiferus under laboratory conditions. Aquacult., 65:363-370.

WICKINS, J.F., BEARD,T.W. 1974. Observations on the breading and growth of the giant freshwater prawn Macrobrachium rosenbergii (de Man) in the laboratory. Aquacult., 3:159-174.

Recebido em: 14/01/99

Aceito em: 14/07/99 